\title{
Effect of COVID-19 on epidemiological characteristics of road traffic injuries in Suzhou: a retrospective study
}

\author{
Wenjuan Huang ${ }^{1+}$, Qi Lin ${ }^{2 \dagger}$, Feng $X^{3}$ and Du Chen ${ }^{1 *}$ (D)
}

\begin{abstract}
Background: To present the new trends in epidemiology of road traffic injuries (RTIS) during the Coronavirus disease 2019 (COVID-19) pandemic in Suzhou.

Methods: Pre-hospital records of RTIs from January to May in 2020 and the same period in 2019 were obtained from the database of Suzhou pre-hospital emergency center, Jiangsu, China. Data were extracted for analysis, including demographic characteristics, pre-hospital vital signs, transport, shock index, consciousness, pre-hospital death. A retrospective study comparing epidemiological characteristics of RTIs in Suzhou during the 5-month period in 2020 to the parallel period in 2019 was performed.

Results: A total of 7288 RTIs in 2020 and 8869 in 2019 met inclusion criteria. The overall volume of RTIs has statistical difference between the 2 years $(p<0.001$ ), with fewer RTIs in 2020 compared with 2019. Electric bicycle related RTIs increased during the pandemic $(2641,36.24 \%$ vs $2380,26.84 \%, p<0.001)$, with a higher incidence of RTIs with disorder of consciousness (DOC) $(7.22 \%$ vs $6.13 \%, p=0.006)$.

Conclusions: Under the impact of COVID-19, the total number of RTIs in Suzhou from January to May 2020 decreased. This observation was coupled with a rise in electric bicycle related injuries and an increase in the incidence of RTIs with DOC.
\end{abstract}

Keywords: COVID-19, Road traffic injuries, Suzhou, Epidemiology

\section{Background}

The Global status report on road safety 2018, launched by WHO in December 2018, highlights that the number of annual deaths resulting from RTIs has reached 1.35 million. The RTIs are the eighth leading cause of death for people of all ages and the leading killer of children and young adults aged 5-29 years [1]. Low- and middleincome countries bear the greatest burden of road traffic fatalities and injuries [1].

\footnotetext{
* Correspondence: sdfyycd@suda.edu.cn

†Wenjuan Huang and Qi Lin contributed equally to this work.

${ }^{1}$ Department of Critical Care Medicine, the First Affiliated Hospital of Soochow University, Suzhou City, Jiangsu Province, China

Full list of author information is available at the end of the article
}

In China, the RTIs are the second cause of all types of injuries that lead to emergency department visits, constituting $21 \%$ of all injuries, and have become the leading cause of injury deaths [2]. The highest mortality of RTIs occurred among young adults aged $20-45$ years, particularly males, and in rural areas [2].

The COVID-19 is caused by the severe acute respiratory syndrome coronavirus 2 (SARS-CoV-2) [3]. This virus is believed to be transmitted by aerosols and/or droplets, and has showed strong infectivity and pathogenicity $[3,4]$. The institution of interventions including traffic restriction, social distancing, lockdowns were the effective strategy against acquiring and spreading COVID-19 [5]. 
The COVID-19 pandemic has significantly affected all walks of life, including the volume and nature of RTIs. There was a significant decrease in the number of road traffic collisions during the COVID-19-related period of societal restrictions and lockdown [6]. Whilst a research has showed trauma admissions decreased in the lockdown phase with an increased incidence of road traffic accidents [7]. A reduction in the number of road traffic accident fatalities was observed during the lockdown period [8-11]. Furthermore, the mandated societal lockdown policies led to reduction in road traffic accidents resulting in non-serious or no injuries but not those resulting in serious or fatal injuries [12]. In addition, Kunal Rajput et al. have showed that the road traffic collisions involving a car significantly reduced during lockdown, conversely, bike-related road traffic collisions significantly increased [13].

Few studies have showed the characteristics of RTIs in Suzhou during the COVID-19 outbreak. The aim of this study was to present the new trends in epidemiology of RTIs in Suzhou during the COVID-19 pandemic.

\section{Methods}

\section{Study design}

In China, the emergency medical service (EMS) system consists of pre-hospital emergency system and hospital emergency department (ED). Pre-hospital emergency treatment refers to the emergency treatment for critically ill patients outside the hospital and plays an irreplaceable role in meeting people's daily emergency needs, providing medical security for major activities and dealing with emergencies and natural disasters [14]. The hospital ED is the core of the EMS system, and it is a professional department dealing with acute and critical treatment, public emergencies, mass trauma events, natural disasters, and collective poisoning events [14].

In this study, the pre-hospital records were obtained from the database of Suzhou pre-hospital emergency center, Jiangsu province, China. Cases were selected after taking into account the inclusion and exclusion criteria (Fig. 1). The RTIs from January to May in 2020 were included for analysis. The RTIs from the same period in 2019 were used as historical control. The variables, including demographic characteristics, pre-hospital vital signs, transport, injury pattern (shock, DOC, pre- hospital death) were collected. Shock was defined as a shock index (pulse rate /systolic blood pressure) $>1$, and DOC is defined as Glasgow Coma Scale (GCS) $<15$ points. A retrospective analysis was performed.

The primary outcome of interest was the trend in the number of RTIs. Secondary outcomes included changes in transport, injury nature, and pre-hospital mortality.

\section{Statistical analysis}

Categorical data were expressed as frequency (percentage), and compared using chi-square test. Continuous variables were tested for normality using Shapiro-Wilk test. All of the continuous variables failed to conform to normality were expressed as median (IQR) and compared using Mann-Whitney test. Statistical analyses were completed with STATA 15.0, the graph was plotted in MS Excel. Statistical significance was defined as a 2sided $P$ value of $<0.05$.

\section{Results}

A total of 7288 RTIs in 2020 and 8869 in 2019 met inclusion criteria (Table 1). The overall volume of RTIs has statistical difference between the 2 years $(p<0.001)$, with fewer RTIs in 2020 compared with 2019. In February of these 2 years, the number of RTIs was lowest. The daily number of RTIs in Suzhou in January-May 2020 and January-May 2019 were respectively showed in Fig. 2. Comparatively, the trend in the number of RTIs reports an overall reduction in 2020 when compared to 2019. The most marked reduction occurred in late January and February 2020 when compared to year 2019.

Electric bicycle related RTIs increased during the pandemic $(2641,36.24 \%$ vs $2380,26.84 \%, p<0.001)$, with a higher incidence of RTIs with DOC $(7.22 \%$ vs $6.13 \%$, $p=0.006$ ).

Compared with the RTIs in 2019, the median age of cases in 2020 was 1 year older $(p=0.045)$. Regarding gender, shock and death, no significant differences were detected between the two time periods $(p=0.050, p=$ 0.508 and $p=0.720$ respectively).

\section{Discussion}

The outbreak of COVID-19 has caused global concerns. Currently, the therapeutics for COVID-19 including supporting treatment, drugs, vaccines, while control and prevention are other strategies to reduce the transmission within China and elsewhere $[15,16]$. The local government in Wuhan announced the suspension of public transportation, including the closure of railway stations, highways and airports on January 23, 2020, to prevent further disease spread [17]. Consequently, Hubei province was placed under lockdown approximately 3 weeks after the start of COVID-19 outbreak [18]. The Chinese government made great efforts to control the flow of people. Shopping malls and other entertainment activities were closed, in-person classes were replaced by online ones, public transport was restricted, public gatherings were banned and routine health checks were carried out in order to prevent the spread of SAR-CoV2 right after Wuhan shutdown [19].

Due to the impact of COVID-19 and relevant control measures, the way people travel has also undergone 
RTIs recorded in the database of Suzhou pre-hospital emergency center

Inclusion criteria:

Cases in January-May 2020 served as observation group

Cases in January-May 2019 served as historical control

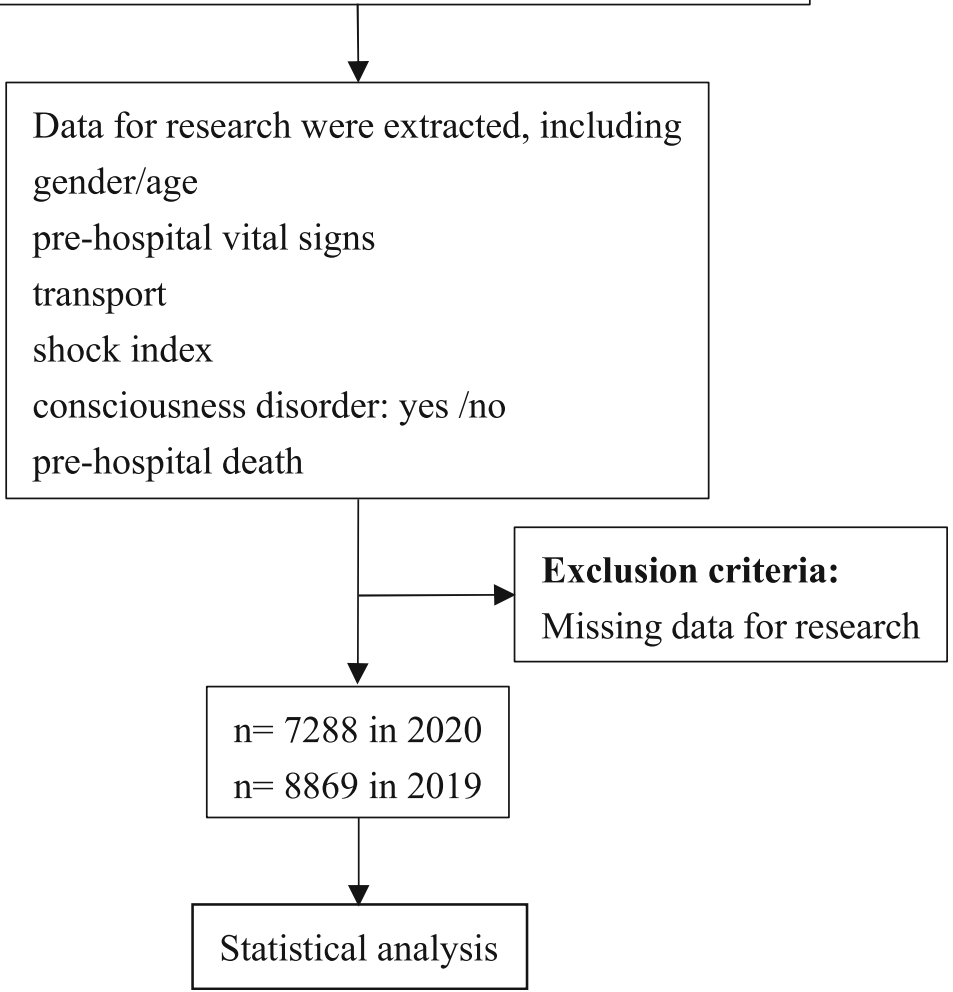

Fig. 1 Methodology of our study-depicting research

subtle changes. Indeed, some new trends have emerged in the epidemiology of RTIs during pandemic in Suzhou.

In the present study, the total volume of RTIs decreased during the COVID-19 pandemic, which is consistent with previous studies $[6,12,13]$. The decline is mostly attributed to the above restrictions. People might be more inclined to perform activities at home which would result in less car traffic and less congestion during peak hours [20]. This could account for reduced traffic burden, resulting in a decrease in the number of RTIs.

Furthermore, in this research, the most marked reduction occurred in late January and February 2020 when compared to year 2019. This is due to the fact that domestic pandemic was most severe in late January and February 2020 and under the strictest control. In February 2019, the volume of RTIs was also the lowest throughout the year. This is attributed to Spring Festival holiday in early February. Large numbers of migrant workers have left Suzhou and returned to hometowns, which resulting in declines in traffic burden and RTIs.

Whilst in this study, electric bicycle related RTIs increased during the pandemic, with a higher incidence of RTIs with DOC. China is a large consumer of electric bicycle. In order to avoid close contact during the pandemic, people preferred electric bicycle to public transport, which directly led to an increase in electric bicycle related RTIs. This result is consistent with that of Kunal Rajput et al. [13]. They have reported an increase in bike- or pushbike related road traffic collisions during lockdown, which is likely due to the fact that riding a bike became the chosen mode of exercise for many people in warmer weather while keeping to the statutory $2 \mathrm{~m}$ distance. In addition, the traffic volume has decreased but the cycling speed may have increased due to lower congestion on roads. 
Table 1 Characteristics of RTIs in Suzhou in January-May 2020 and January-May 2019

\begin{tabular}{|c|c|c|c|}
\hline & $\begin{array}{l}2019 \\
(n=8869)\end{array}$ & $\begin{array}{l}2020 \\
(n=7288)\end{array}$ & $p$ value \\
\hline Number of RTIs, $n$ (\%) & & & $<0.001$ \\
\hline Jan. & 1834(20.68) & 1462(20.06) & \\
\hline Feb. & $996(11.23)$ & $475(6.52)$ & \\
\hline Mar. & $1831(20.65)$ & $1452(19.92)$ & \\
\hline Apr. & 2073(23.37) & 1777(24.38) & \\
\hline May & $2135(24.07)$ & 2122(29.12) & \\
\hline Electric bicycle related RTIs, $\mathbf{n}(\%)$ & $2380(26.84)$ & $2641(36.24)$ & $<0.001$ \\
\hline Gender & & & 0.050 \\
\hline Female, n (\%) & 4088(46.09) & $3247(44.55)$ & \\
\hline Male, n (\%) & $4781(53.91)$ & $4041(55.45)$ & \\
\hline Age, years (SD) & $47(27)$ & $48(27)$ & 0.045 \\
\hline \multicolumn{4}{|l|}{ Vital signs } \\
\hline $\mathrm{PR}$, bpm (SD) & $83(14)$ & $82(15)$ & 0.025 \\
\hline RR, bpm (SD) & 18(3) & $18(2)$ & 0.385 \\
\hline $\mathrm{SBP}, \mathrm{mmHg}(\mathrm{SD})$ & $133(28)$ & $134(28)$ & 0.436 \\
\hline $\mathrm{DBP}, \mathrm{mmHg}(\mathrm{SD})$ & $82(17)$ & $81(17)$ & 0.248 \\
\hline \multicolumn{4}{|l|}{ Injuries } \\
\hline Shock, n (\%) & $245(2.76)$ & $214(2.94)$ & 0.508 \\
\hline DOC, n (\%) & $544(6.13)$ & $526(7.22)$ & 0.006 \\
\hline Death, n (\%) & $91(1.03)$ & $79(1.08)$ & 0.720 \\
\hline
\end{tabular}

Abbreviations: $R T I s$ road traffic injuries, $P R$ pulse rate, $R R$ respiratory rate, $b p m$ beats per minute, $S B P$ systolic blood pressure, $D B P$ diastolic blood pressure, $S D$ standard deviation, DOC disorder of consciousness
Meanwhile, in the event of road traffic accidents, riders without wearing helmets are vulnerable to head injury [21, 22]. Generally, in road traffic collisions, the DOC is often associated with head injury. During the study period, electric bicycle riders in Suzhou generally did not wear helmets, resulting in a rise in the incidence of RTIs with DOC. Therefore, Suzhou government has carried out the "helmet-belt" safety protection activity, to increase the use of helmets among electric bicycle riders and seatbelts among car occupants, which is very necessary for electric bicycle riders to reduce head injury.

In the present study, the mortality of RTIs reported in January-May 2020 was similar to that reported during the parallel period in 2019. This result is inconsistent with those reported in previous studies [9-11]. In the above studies, fatalities resulting from road traffic accidents exhibit a significant decrease, possibly because of the restrictions (such as lockdowns) that were imposed to contain the spread of the COVID-19 pandemic in 2020 [9-11]. In addition, the deaths referred in our study were those occurring in pre-hospital, deaths occurring in hospitals were not included, which might lead to underestimated fatalities resulting from RTIs.

Compared with the RTIs in 2019, the median age of cases in 2020 was 1 year older $(p=0.045)$. This finding deserves further study when more data will become available. Concerning gender and shock, our analysis did not reveal any statistically significant differences.

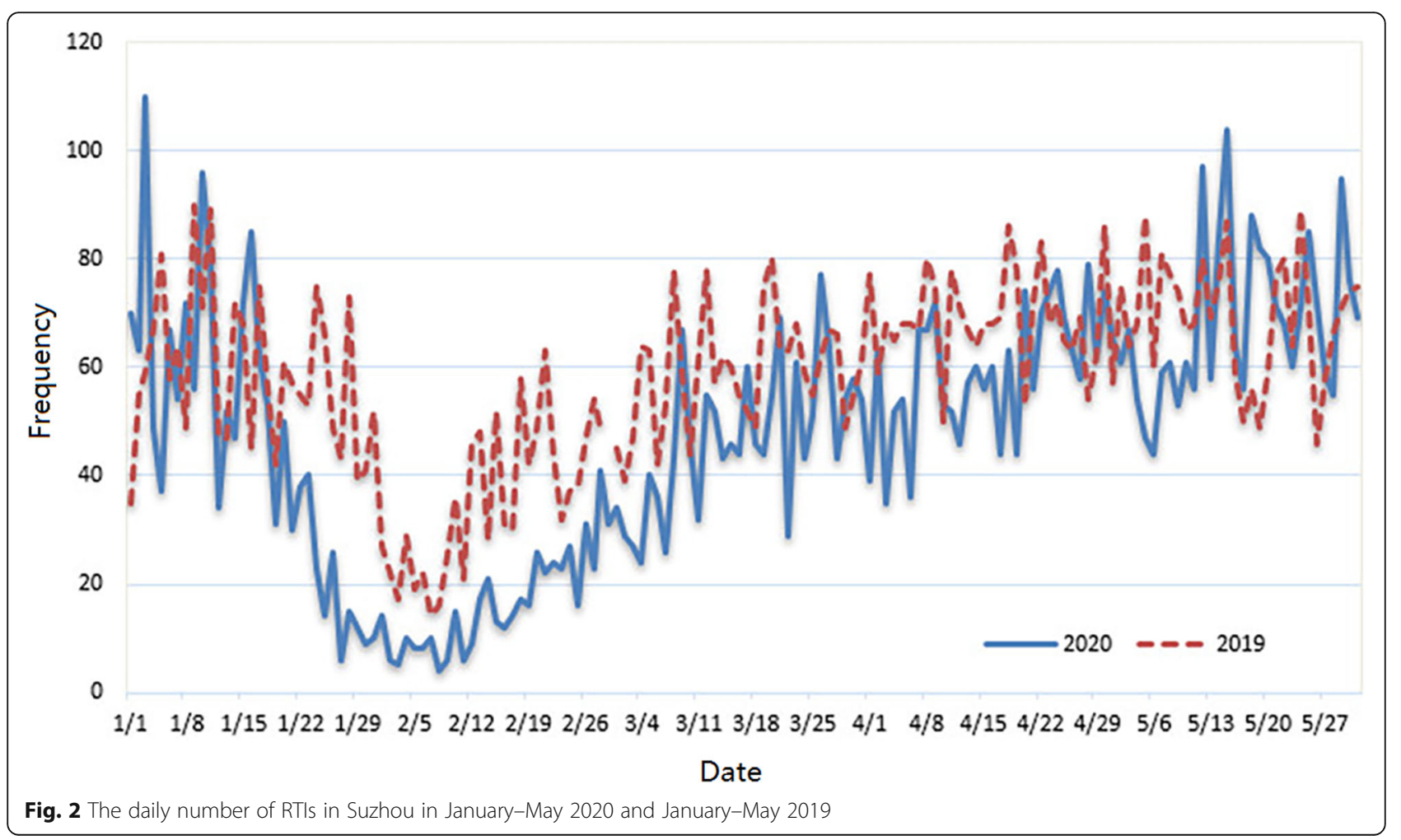


The present study has some limitations. First, only the data of the 5 months from January to May were analyzed, but the medium - and long-term impacts of COVID-19 epidemic on RTIs were not clear. Second, Suzhou is a prefecture-level city in China, the involvement of just one center resulted in a smaller sample size. This study could be repeated with a larger sample size, which would add weight to our conclusions. Third, there are many factors affecting RTIs in reality, the influence of other confounding factors cannot be completely excluded from the historical data only. In the cases of minor injuries, emergency services may not be activated and therefore no pre-hospital records are available. The present study potentially underestimates the true number of RTIs.

\section{Conclusions}

Under the impact of COVID-19, the total number of RTIs in Suzhou from January to May 2020 decreased. This observation was coupled with a rise in electric bicycle related injuries and an increase in the incidence of RTIs with DOC.

\section{Abbreviations}

RTIs: road traffic injuries; COVID-19: Coronavirus disease 2019; SARS-CoV2: severe acute respiratory syndrome coronavirus 2; EMS: emergency medical service; ED: emergency department; GCS: Glasgow Coma Scale; PR: pulse rate; RR: respiratory rate; bpm: beats per minute; SBP: systolic blood pressure; DBP: diastolic blood pressure; SD: standard deviation; DOC: disorder of consciousness

\section{Supplementary Information}

The online version contains supplementary material available at https:/doi. org/10.1186/s12873-021-00483-7.

\section{Additional file 1.}

\section{Acknowledgements}

Not applicable.

\section{Authors' contributions}

DC conceived the study, and designed the protocol. QL and DC collected and analysed the data. WJH drafted the manuscript. WJH and DC contributed substantially to its revision. FX supervised the conduct of the study and data collection. All authors read and approved the final manuscript.

\section{Funding}

No funding was received.

\section{Availability of data and materials}

All data generated or analyzed during this study are included in this article and its supplementary information files.

\section{Declarations}

\section{Ethics approval and consent to participate}

This study was approved by the Ethics Committee of the First Affiliated Hospital of Soochow University. The approval number is 2020226. All patient records were anonymized and de-identified and the Ethics Committee waived the need for written, informed consent before analysis due to the retrospective nature of the data.

\section{Consent for publication}

Consent for publication of raw data not obtained but dataset is fully anonymous in a manner that can easily be verified by any user of the dataset. Publication of the dataset clearly and obviously presents minimal risk to confidentiality of study participants.

\section{Competing interests}

The authors declare that they have no competing interests.

\section{Author details}

'Department of Critical Care Medicine, the First Affiliated Hospital of Soochow University, Suzhou City, Jiangsu Province, China. ${ }^{2}$ Suzhou Emergency Center, Suzhou City, Jiangsu Province, China. ${ }^{3}$ Department of Emergency Medicine, the First Affiliated Hospital of Soochow University, Suzhou City, Jiangsu Province, China.

Received: 15 November 2020 Accepted: 19 July 2021

Published online: 26 July 2021

\section{References}

1. Global status report on road safety 2018. Geneva: World Health Organization; 2018. Licence: CC BY-NC-SA 3.0 IGO. https://www.who.int/ publications/i/item/9789241565684. Accessed: March 20, 2021.

2. Ma S, Li QF, Zhou MG, Duan LL, Bishai D. Road traffic injury in China: a review of National Data Sources. Traffic Injury Prev. 2012;13(S1):57-63. https://doi.org/10.1080/15389588.2011.633945.

3. Salian VS, Wright JA, Vedell PT, Nair S, Li C, Kandimalla M, et al. COVID-19 transmission, current treatment, and future therapeutic strategies. Mol Pharm. 2021;18(3):754-71. https://doi.org/10.1021/acs.molpharmaceut. 0c00608.

4. Pascarella G, Strumia A, Piliego C, Bruno F, Del Buono R, Costa F, et al. COVID-19 diagnosis and management: a comprehensive review. J Intern Med. 2020;288(2):192-206. https://doi.org/10.1111/joim.13091.

5. Pan A, Liu L, Wang C, Guo H, Hao X, Wang Q, et al. Association of Public Health Interventions with the epidemiology of the COVID-19 outbreak in Wuhan, China. J Am Med Assoc. 2020;323(19):1915-23. https://doi.org/10.1 001/jama.2020.6130.

6. Jacob S, Mwagiru D, Thakur I, Moghadam A, Oh T, Hsu J. Impact of societal restrictions and lockdown on trauma admissions during the COVID-19 pandemic: a single-Centre cross-sectional observational study. ANZ J Surg. 2020;90(11):2227-31. https://doi.org/10.1111/ans.16307.

7. Ajayi B, Trompeter A, Arnander M, Sedgwick P, Lui DF. 40 days and 40 nights: clinical characteristics of major trauma and orthopaedic injury comparing the incubation and lockdown phases of COVID-19 infection. Bone Joint Open. 2020;7(1):330-8.

8. Calderon-Anyosa RJC, Kaufman JS. Impact of COVID-19 lockdown policy on homicide, suicide, and motor vehicle deaths in Peru. Prev Med. 2021;143: 106331. https://doi.org/10.1016/j.ypmed.2020.106331.

9. Khurshid A, Sohail A, Khurshid M, Shah MU, Jaffry AA. Analysis of Road Traffic Accident Fatalities in Karachi, Pakistan: An Autopsy-Based Study. Cureus. 13(4):e14459.

10. Fahy S, Moore J, Kelly M, Flannery O, Kenny P. Analysing the variation in volume and nature of trauma presentations during COVID-19 lockdown in Ireland. Bone Joint Open. 2020;6(1):261-6.

11. Sakelliadis El, Katsos KD, Zouzia El, Spiliopoulou CA, Tsiodras S. Impact of Covid-19 lockdown on characteristics of autopsy cases in Greece. Comparison between 2019 and 2020. Forensic Sci Int. 2020;313:110365.

12. Qureshi Al, Huang W, Khan S, Lobanova I, Siddiq F, Gomez CR, et al. Mandated societal lockdown and road traffic accidents. Accid Anal Prev. 2020;146:105747. https://doi.org/10.1016/j.aap.2020.105747.

13. Rajput K, Sud A, Rees M, Rutka O. Epidemiology of trauma presentations to a major trauma Centre in the north west of England during the COVID-19 level 4 lockdown. Eur J Trauma Emerg Surg. 2021;47(3):631-6. https://doi. org/10.1007/s00068-020-01507-w.

14. Shi $X$, Bao J, Zhang H, Wang H, Wang $Y$, Li L, et al. Emergency medicine in China: a review of the history of progress and current and future challenges after 40 years of reform. Am J Emerg Med. 2020;38(3):662-9. https://doi. org/10.1016/j.ajem.2019.11.008.

15. Huilan T, Sheng T, Gao S, Shao A, Sheng J. The epidemiological and clinical features of COVID-19 and lessons from this global infectious public health event. Infection. 2020. 
16. Ahn DG, Shin HJ, Kim MH, Lee S, Kim HS, Myoung J, et al. Current status of epidemiology, diagnosis, therapeutics, and vaccines for novel coronavirus disease 2019 (COVID-19). J Microbiol Biotechnol. 2020;30(3):313-24. https:// doi.org/10.4014/jmb.2003.03011.

17. Announcement from the Headquarter for novel coronavirus pneumonia prevention and control (No 1). Beijing: China National Health Commission. http://www.gov.cn/xinwen/2020-01/23/content_5471751.htm [March 22, 2020].

18. Lau H, Khosrawipour V, Kocbach P, Mikolajczyk A, Schubert J, Bania J, et al. The positive impact of lockdown in Wuhan on containing the COVID-19 outbreak in China. J Travel Med. 2020;27(3).

19. Liu NN, Tan JC, Li J, Li S, Cai Y, Wang H. COVID-19 pandemic: experiences in China and implications for its prevention and treatment worldwide. Curr Cancer Drug Targets. 2020;20(6):410-6. https://doi.org/10.2174/156800962 0666200414151419.

20. De Vos J. The effect of COVID-19 and subsequent social distancing on travel behavior. Trans Res Interdisc Perspec. 2020;5:100121. https://doi.org/10.1016/ j.trip.2020.100121.

21. Gomei $\mathrm{S}$, Hitosugi $\mathrm{M}$, Ikegami $\mathrm{K}$, Tokudome $\mathrm{S}$. Assessing injury severity in bicyclists involved in traffic accidents to more effectively prevent fatal bicycle injuries in Japan. Med Sci Law. 2013;53(4):194-8. https://doi.org/1 $0.1177 / 0025802413481011$

22. Bíl M, Dobiáš M, Andrášik R, Bílová M, Hejna P. Cycling fatalities: when a helmet is useless and when it might save your life. Saf Sci. 2018;105:71-6. https://doi.org/10.1016/j.ssci.2018.02.005.

\section{Publisher's Note}

Springer Nature remains neutral with regard to jurisdictional claims in published maps and institutional affiliations.

Ready to submit your research? Choose BMC and benefit from:

- fast, convenient online submission

- thorough peer review by experienced researchers in your field

- rapid publication on acceptance

- support for research data, including large and complex data types

- gold Open Access which fosters wider collaboration and increased citations

- maximum visibility for your research: over $100 \mathrm{M}$ website views per year

At $\mathrm{BMC}$, research is always in progress.

Learn more biomedcentral.com/submissions 